\title{
Robotic-assisted Total Hip Arthroplasty May Reduce the Risk of Complications in Patients Younger than 35
}

\author{
David Novikov BS ${ }^{1}$, Tyler Luthringer MD ${ }^{1}$, Zlatan Cizmic $\mathrm{MD}^{2}$, Siddharth \\ Mahure MD MBA ${ }^{1}$, Nima Eftekhary MD ${ }^{1}$, Roy Davidovitch $\mathrm{MD}^{1}$, and \\ Jonathan Vigdorchik MD ${ }^{3}$ \\ ${ }^{1}$ NYU Langone Orthopedic Hospital \\ ${ }^{2}$ Ascension Providence Hospital \\ ${ }^{3}$ Hospital for Special Surgery \\ davenovikov@gmail.com, Tyler.Luthringer@nyulangone.org, \\ zlatancizmic91@gmail.com, siddharth.mahure@nyulangone.org, \\ nima.eftekhary@nyulangone.org, roy.davidovitch@nyulangone.org, \\ vigdorchikj@hss.edu
}

\begin{abstract}
INTRODUCTION: In younger patients during total hip arthroplasty (THA), the presence of morphologic deformities, previous surgeries, and retained hardware, can pose technical challenges making reconstruction difficult. The purpose of our study is to assess the outcome of robotic-assisted THA compared to conventional THA in patients younger than 35 years old.
\end{abstract}

METHODS: A retrospective analysis of 123 patients younger than 35 years old who underwent primary unilateral THA between January 2013 and April 2018 was conducted. Patients were divided into two cohorts: (1) robotic-assisted THA (r-THA) and (2) conventional-THA (c-THA). Demographics, operative details, and post-operative outcomes were carefully studied. Radiographic analysis included measurement of postoperative acetabular anteversion and inclination angles as well as perioperative leg length discrepancies.

RESULTS: Of the total 123 patients, 30 patients (32 hips) were in the robotic-THA cohort, and 93 patients (100 hips) were in the c-THA cohort. Patients in the r-THA cohort were younger $(26.6 \pm 6.2$ vs. $29.0 \pm 5.3 ; p=0.03)$ and had a higher mean BMI $(29.8 \pm 8.2$ vs. $25.7 \pm 5.9 ; p=0.03)$ at surgery. The most common indications for THA were DDH and osteonecrosis. The acetabular component was positioned within Lewinnek's safe zone more often in the r-THA cohort compared to the c-THA cohort $(94 \%$ vs $65 \%$; $p<0.01)$. Patients in the c-THA group were more likely to experience clinically significant higher 
rates of dislocation ( $2.9 \%$ vs. 0 ), revision $(6.8 \%)$, any postoperative complication $(7.8 \%)$, and 90-day readmission (2.9) following THA.

CONCLUSION: Robotic THA can help improve outcomes in younger THA recipients. Future studies with larger cohorts and longer follow-up times should evaluate outcomes in this historically technically demanding patient population.

\section{Introduction}

Total hip arthroplasty (THA) is considered to be one of the most successful surgical procedures offered to patients in the last century. Concurrent with its success, indications for THA have been increasingly expanded to include younger patients with end-stage degenerative disease. ${ }^{1-3}$ In younger patients during THA, the presence of morphologic deformities, limb length discrepancies (LLD), previous surgeries, and retained hardware, can pose technical challenges making reconstruction difficult. ${ }^{4-6}$

Robotic-assisted platforms were first introduced to total joint arthroplasty approximately two decades ago in an effort to enhance clinical outcomes through improved component alignment and the restoration of optimal joint kinematics. ${ }^{7}$ Robotic-assisted THA (r-THA) can be especially useful in cases where there is an absence of normal anatomic landmarks as the technology can help account for anatomic variances through its image-based preoperative planning capabilities. ${ }^{7}$ The purpose of our study is to assess the outcomes of r-THA compared to conventional THA in patients younger than 35 years old. Our hypothesis is that r-THA will have improved radiographic outcomes, but no difference in clinical outcomes or complications compared to conventional THA in patients under the age of 35 . 


\section{Methods \& Materials}

\subsection{Data Collection}

A retrospective analysis of 123 consecutive patients younger than 35 years old that underwent primary unilateral THA between January 2013 and April 2018 at a large, urban, academic, tertiary care center was conducted. Patients undergoing simultaneous bilateral THA or revision THA were excluded from the search. Patients were divided into two cohorts: (1) robotic-assisted THA (r-THA) and (2) conventional-THA (c-THA). All cases in the r-THA cohort were performed using the MAKO ${ }^{\mathrm{TM}}$ robotic hip system $\left(\mathrm{MAKO}^{\mathrm{TM}}\right.$ Surgical Corporation, Fort Lauderdale, FL, USA). Baseline patient characteristics, surgical characteristics, and quality outcomes up to the patient's last follow-up were recorded.

\subsection{Radiographic Analysis}

Acetabular cup version and inclination angles were evaluated on standardized preoperative anteroposterior (AP) pelvis radiographs for all cases. Cup orientation was deemed within Lewinnek's "safe zone" if it had an anteversion of $15^{\circ}+/-10^{\circ}$ and inclination $40^{\circ}+/-10^{\circ} .{ }^{8}$ Leg length discrepancies were measured as the difference in distance from a horizontal line at the inferior aspect of each hemipelvis teardrop to the most prominent point of the lesser trochanter on standard postoperative AP pelvis radiographs. ${ }^{9}$

\subsection{Statistical Analysis}

All descriptive and inferential statistics were conducted using SPSS v23 (International Business Machines, Armonk NY) statistics software. Chi-square tests and Fishers exact tests were performed to compare categorical variables and two-tailed Student's t-tests were performed to compare means among continuous variables. All tests performed were 2 -sided where a p-value $<0.05$ was deemed statistically significant.

\section{Results}

\subsection{Patient and Surgical Characteristics}

Of the total 123 patients, 30 patients ( 32 hips) were in the r-THA cohort, and 93 patients (100 hips) were in the c-THA cohort. Patients in the r-THA cohort were younger (26.6 \pm 6.2 vs. 29.0 \pm 5.3 ; $p=0.03)$ and had a higher mean BMI (29.8 \pm 8.2 vs. $25.7 \pm 5.9 ; p=0.03)$ at surgery.

Patients in the r-THA cohort had significantly longer mean operative times than patients in the cTHA cohort $(138.7 \pm 34.0$ minutes vs. $107.1 \pm 46.7$ minutes; $p<0.01)$. There was a significantly higher rate of modular prosthesis implantation in the r-THA cohort compared to the c-THA cohort (r-THA: $\mathrm{n}=11,34.4 \%$ vs. c-THA: $\mathrm{n}=5,5.0 \%$; $\mathrm{p}<0.01$ ).

Radiographic analysis revealed a greater mean radiographic LLD correction in the r-THA cohort (r-THA: $8.7 \pm 7.2$ vs. $c$-THA: $5.7 \pm 6.0 \mathrm{~mm}$; $\mathrm{p}<0.05$ ). The acetabular component was positioned within 
Robotic-assisted Total Hip Arthroplasty May Reduce the Risk of Complications ... D. Novikov et al.

Lewinnek's safe zone more often in the r-THA cohort compared to the c-THA cohort (94\% vs $65 \%$; $\mathrm{p}<0.01$ ) (Figure 1). The remainder of the characteristics were statistically similar (Table 1).

\begin{tabular}{|c|c|c|c|}
\hline Table 1. Patient and Surgical Characteristics & $\begin{array}{l}\text { MAKO-THA } \\
\text { n= 30 patients } \\
32 \text { hips }(\%)\end{array}$ & $\begin{array}{l}\text { Conventional-THA } \\
\text { n=93 patients } \\
100 \text { hips }(\%)\end{array}$ & $p$-value \\
\hline Age (yrs.) (mean \pm SD [range]) & $26.6 \pm 6.2[13-35]$ & $29.0 \pm 5.3[15-35]$ & 0.03* \\
\hline Gender & & & 0.94 \\
\hline Male & $11(38)$ & $37(40)$ & \\
\hline Female & $19(62)$ & $56(60)$ & \\
\hline ASA $($ mean \pm SD [range]) & $1.9 \pm 0.6[1-3]$ & $1.9 \pm 0.6[1-3]$ & 0.86 \\
\hline BMI $\left(\mathrm{kg} / \mathrm{m}^{2}\right)($ mean \pm SD [range] $)$ & $29.8 \pm 8.2[15.1-54.3]$ & $25.7 \pm 5.9[16.6-40.4]$ & $0.03 *$ \\
\hline Indication for THA & & & 0.12 \\
\hline Osteonecrosis & $8(26)$ & $46(46)$ & \\
\hline DDH & $14(45)$ & $26(26)$ & \\
\hline Crowe & & & 0.26 \\
\hline $\mathbf{I}$ & $6(42.9)$ & $10(38.4)$ & \\
\hline II & $7(50)$ & $7(26.9)$ & \\
\hline III \& IV & $1(7.1)$ & $6(23.0)$ & \\
\hline $\mathbf{O A}$ & $5(16)$ & $10(10)$ & \\
\hline Other $\uparrow$ & $4(12.5)$ & $21(21)$ & \\
\hline Previous hip surgery (Yes) & $10(33.3)$ & $25(27)$ & 0.94 \\
\hline Length of follow-up (months) (mean $\pm \mathrm{SD}$ [range]) & $19.3 \pm 13.9[3-43]$ & $23.9 \pm 18.3[3-65]$ & 0.14 \\
\hline Anesthesia Method & & & 0.10 \\
\hline Spinal & $13(40)$ & $62(62)$ & \\
\hline General & $19(60)$ & $38(38)$ & \\
\hline Surgical time (minutes) (mean $\pm \mathrm{SD}$ [range]) & $138.7 \pm 34.0$ & $107.1 \pm 46.7$ & $<0.01 *$ \\
\hline Intraoperative ROH (Yes) & $2(6.3)$ & $2(2)$ & 0.22 \\
\hline Intraoperative subtrochanteric osteotomy & $1(3.1)$ & $0(0)$ & 0.24 \\
\hline Use of modular femoral prosthesis (Yes) & $11(34.3)$ & $5(5)$ & $<0.01 *$ \\
\hline Hospital LOS (days) (mean \pm SD [range]) & $3.0 \pm 1.4[1-6]$ & $2.6 \pm 1.4[0-6]$ & 0.44 \\
\hline Preoperative LLD $(\mathrm{mm})($ mean \pm SD $[$ range] $)$ & $11.9 \pm 9.5[0.3-33.7]$ & $9.3 \pm 11.3[0-85]$ & 0.28 \\
\hline Postoperative LLD $(\mathrm{mm})($ mean $\pm \mathrm{SD}$ [range] $)$ & $7.2 \pm 6[0.3-20.3]$ & $7.6 \pm 12.1[0.8-63]$ & 0.90 \\
\hline LLD correction $\ddagger(\mathrm{mm})($ mean $\pm \mathrm{SD}[$ range $])$ & $8.7 \pm 7.2[0.5-28.1]$ & $5.7 \pm 6.0[0-24.1]$ & $<0.05^{*}$ \\
\hline Cup Positioned within Lewinnek Safe Zone & & & $<0.01 *$ \\
\hline Yes & $30(94)$ & $65(65)$ & \\
\hline No & $2(6)$ & $35(35)$ & \\
\hline
\end{tabular}


Discharge Disposition

Home

Acute rehab

Skilled nursing facility

* Statistically significant

†Other includes Legg-Calvé-Perthes, rheumatoid arthritis, septic arthritis, juvenile rheumatoid arthritis, and trauma

†Preoperative LLD - Postoperative LLD

\subsection{Postoperative Outcomes}

The mean follow-up time was similar in both cohorts (r-THA: 19.3 \pm 13.9 months vs. c-THA: $23.9 \pm 18.3$ months; $p=0.14)$. Patients in the c-THA group were more likely to experience clinically significant higher rates of dislocation $(2.9 \%$ vs. $0 \%)$, revision surgery $(6.8 \%$ vs. $0 \%)$, any postoperative

\begin{tabular}{|c|c|c|c|}
\hline Table 2 Postoperative Outcomes & $\begin{array}{l}\text { MAKO-THA } \\
\text { n=30 patients } \\
32 \text { hips }(\%)\end{array}$ & $\begin{array}{l}\text { Conventional-THA } \\
\text { n=93 patients } \\
100 \text { hips (\%) }\end{array}$ & $p$-value \\
\hline Dislocation & $0(0)$ & $3(2.9)$ & 0.33 \\
\hline Revision THA & $0(0)$ & $7(6.8)$ & 0.14 \\
\hline Instability & - & 2 & \\
\hline Infection & - & 2 & \\
\hline Aseptic Loosening & - & 2 & \\
\hline Periprosthetic fracture & & 1 & \\
\hline $\begin{array}{l}\text { Any postoperative surgical } \\
\text { complication }\end{array}$ & $0(0)$ & $8(7.8)$ & 0.10 \\
\hline 90-day readmission & $0(0)$ & $3(2.9)$ & 0.33 \\
\hline
\end{tabular}

Figure 1. Cup Positioning with Conventional and Robotic Total Hip Arthroplasty Scatter Plot

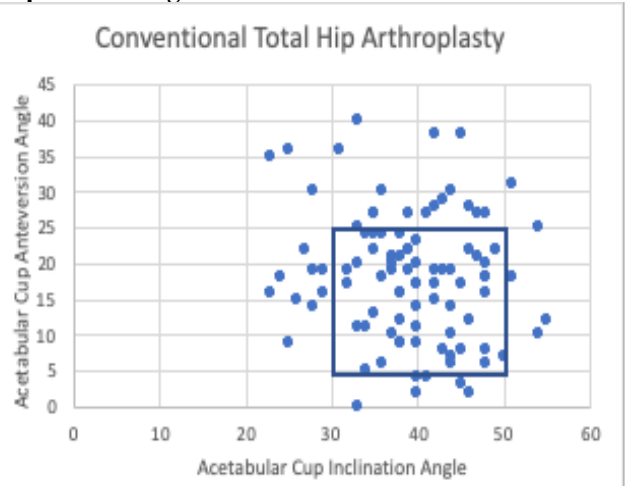
MAKO-Assisted Total Hip Arthroplasty

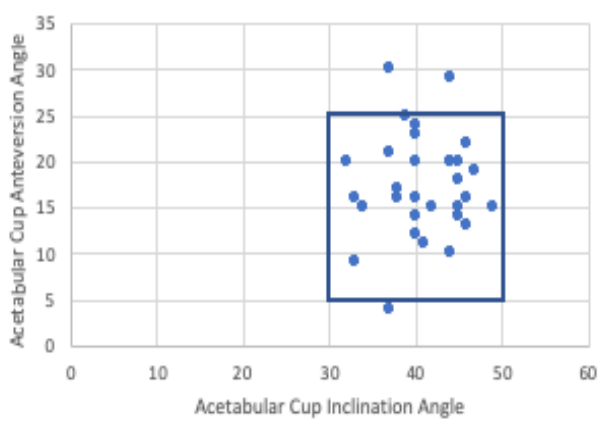


complication ( $7.8 \%$ vs. $0 \%$ ), and 90 -day readmission $(2.9 \%$ vs. $0 \%)$ following THA. However, these differences in outcome rates failed to reach statistical significance (Table 2).

\section{Discussion}

The findings of the present study demonstrate that rates of dislocation, revision THA, and all-cause postoperative surgical complications favored the use of robotic-assisted THA in this young adult population. Moreover, patients in the r-THA cohort had acetabular cups positioned within the safe zone significantly more often than patients in the c-THA cohort. This finding is in accordance with a previous study by Kamara et al that found that the use of robotic-THA is associated with a statistically higher percentage of acetabular cups placed within the surgeon's target zone $(97 \%)$ when compared to conventional posterior $(76 \%)$ and fluoroscopic anterior $(84 \%)$ THA approaches $(p<0.01) .{ }^{10}$

Regarding LLD, the present study demonstrates that there was a significantly larger LLD correction achieved in the r-THA cohort when compared to the c-THA cohort (r-THA: 8.7 vs. c-THA: 5.7; $\mathrm{p}<0.05$ ). This finding is in contrast to a recent study by Kayani et al. in which the authors demonstrated no difference in the accuracy of correcting LLD between c-THA and r-THA recipients. ${ }^{11}$ It is possible that the higher rate of DDH in the r-THA cohort ( $45 \%$ vs. $25 \%)$ increased the mean preoperative LLD resulting in a greater mean LLD correction.

The mean operative time was longer in the r-THA cohort despite a similar complexity of cases among both cohorts, as defined by various patient characteristics (Crowe classification) and intraoperative variables (i.e. removal of hardware, subtrochanteric osteotomy). This finding is in accordance with a recent meta-analysis by Chen et al. that demonstrated a trend towards longer mean surgical times with robotic-assisted THA, with these cases taking 23.21 minutes longer than conventional THA, however this failed to reach statistical significance $(p=0.09){ }^{12}$

The present study is not without limitations. As a retrospective study, selection bias could have been introduced into the study, especially considering the differences in age and BMI among both cohorts. While it is possible that the outcome trends of the present study can reach a statistical significance with a larger sample size, it would be very difficult to capture a cohort of the desired age group at that magnitude. Additionally, there is a surgeon-specific bias that results from a different number of surgeons performing cases among the cohorts, possibly confounding the results.

\section{Conclusion}

Robotic-assisted THA appears to limit early dislocations, revisions, and hospital readmissions within the first two postoperative years for THA-recipients under 35 years of age. This young adult population will continue to be followed to evaluate for differences in long-term survivorship and outcomes between r-THA and c-THA. Future studies with larger cohorts and longer follow-up times should evaluate outcomes in this historically technically demanding patient population.

\section{References}

1. Springer, B. D. et al. Cementless Femoral Components in Young Patients: Review and MetaAnalysis of Total Hip Arthroplasty and Hip Resurfacing. J. Arthroplasty 24, $2-8$ (2009).

2. Pakos, E. E., Paschos, N. K. \& Xenakis, T. A. Long Term Outcomes of Total Hip Arthroplasty in Young Patients under 30. Arch. bone Jt. Surg. 2, 157-62 (2014).

3. Wangen, H., Lereim, P., Holm, I., Gunderson, R. \& Reikerås, O. Hip arthroplasty in patients younger than 30 years: excellent ten to 16-year follow-up results with a HA-coated stem. 
doi:10.1007/s00264-006-0309-2

4. Clohisy, J. C. et al. Function and Fixation of Total Hip Arthroplasty in Patients 25 Years of Age or Younger. Clin. Orthop. Relat. Res. 468, 3207-3213 (2010).

5. Learmonth, I. D., Heywood, A. W., Kaye, J. \& Dall, D. Radiological loosening after cemented hip replacement for juvenile chronic arthritis. J. Bone Joint Surg. Br. 71, 209-12 (1989).

6. Polkowski, G. G., Callaghan, J. J., Mont, M. A. \& Clohisy, J. C. Total Hip Arthroplasty in the Very Young Patient. J. Am. Acad. Orthop. Surg. 20, 487-497 (2012).

7. Jacofsky, D. J. \& Allen, M. Robotics in Arthroplasty: A Comprehensive Review. Journal of Arthroplasty (2016). doi:10.1016/j.arth.2016.05.026

8. Lewinnek, G. E., Lewis, J. L., Tarr, R., Compere, C. L. \& Zimmerman, J. R. Dislocations after total hip-replacement arthroplasties. J. Bone Joint Surg. Am. 60, 217-20 (1978).

9. Woolson, S. T., Hartford, J. M. \& Sawyer, A. Results of a method of leg-length equalization for patients undergoing primary total hip replacement. J. Arthroplasty 14, 159-164 (1999).

10. Kamara, E., Robinson, J., Bas, M. A., Rodriguez, J. A. \& Hepinstall, M. S. Adoption of Robotic vs Fluoroscopic Guidance in Total Hip Arthroplasty: Is Acetabular Positioning Improved in the Learning Curve? J. Arthroplasty 32, 125-130 (2017).

11. Kayani, B., Konan, S., Thakrar, R. R., Huq, S. S. \& Haddad, F. S. Assuring the long-term total joint arthroplasty. Bone Joint J. 101-B, 11-18 (2019).

12. Chen, X. et al. Robotic-assisted compared with conventional total hip arthroplasty: systematic review and meta-analysis. Postgrad. Med. J. 94, 335-341 (2018). 